\title{
Calibration of a rule-based intelligent network simulation model
}

\author{
A. A. Memon' ${ }^{1}$ M. Meng' ${ }^{2}$ Y. D. Wong ${ }^{3}$ S. H. Lam $^{4}$
}

Received: 23 March 2015/Revised: 9 November 2015/Accepted: 11 November 2015/Published online: 22 January 2016

(C) The Author(s) 2016. This article is published with open access at Springerlink.com

\begin{abstract}
This paper is focused on calibration of an intelligent network simulation model (INSIM) with reallife transportation network to analyse the INSIM's feasibility in simulating commuters' travel choice behaviour under the influence of real-time integrated multimodal traveller information (IMTI). A transportation network model for the central and western areas of Singapore was simulated in PARAMICS and integrated with INSIM expert system by means of an application programming interface to form the INSIM. Upon calibration, INSIM was able to realistically present complicated scenarios in which real-time IMTI was provided to commuters and the network performance measures being recorded.
\end{abstract}

Keywords Traffic simulation - Integrated traveller information $\cdot$ Calibration $\cdot$ Mode choice

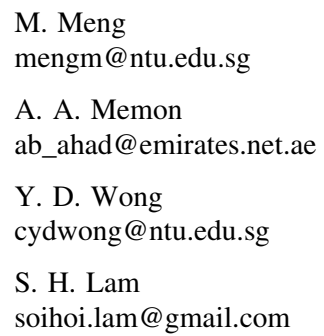

1 IVES (International Vision for Engineering Solutions), Abu Dhabi, United Arab Emirates

2 TUM CREATE, 1 CREATE Way \#10-02 CREATE Tower, Singapore 138602, Singapore

3 School of Civil and Environmental Engineering, Centre for Infrastructure Systems, Nanyang Technological University, Singapore 639798, Singapore

4 Transportation Infrastructure Office, Macao S.A.R., China

\section{Introduction}

Empirical findings of travel behaviour studies have provided evidence that commuters presented with integrated multimodal traveller information (IMTI) in congested travel environment, exhibited a certain degree of mode switching [1-4]. However, from the transportation system's perspective, the potential efficiency and success of various policy measures for eliminating or reducing traffic problems in metropolitan areas largely depend on the level of public acceptance of the traveller information [5-7]. A variety of travel behaviour models based on revealed and stated preference surveys have been developed to study the nature and dynamics of traveller's choice behaviour [811]. Despite the obvious need for assessing user acceptance and the potential impacts of these systems in terms of improving traffic conditions for individual drivers and the overall transportation system, there is still a lack of models to evaluate their full impacts. The idealised analytical models are not appropriate for observing commuters' behaviour dynamics as they lack the ability of representing decision-making processes occurring in a complex dynamic environment. On the other hand, treating travel behaviour within the framework of discrete choice models may not be that effective because, with the spatial and temporal dimensions, the choice set becomes astronomically large if one wishes to gain adequate levels of precision in forecasts. Furthermore, these tests and experiments are very expensive to conduct and do not allow for effective evaluation of different alternatives. Realising the limitations of other approaches with respect to the dynamics of travellers' behaviour under the influence of real-time information, several researches have invested considerable effort in laboratory experiments being conducted with the support of the simulation models [12-16]. 


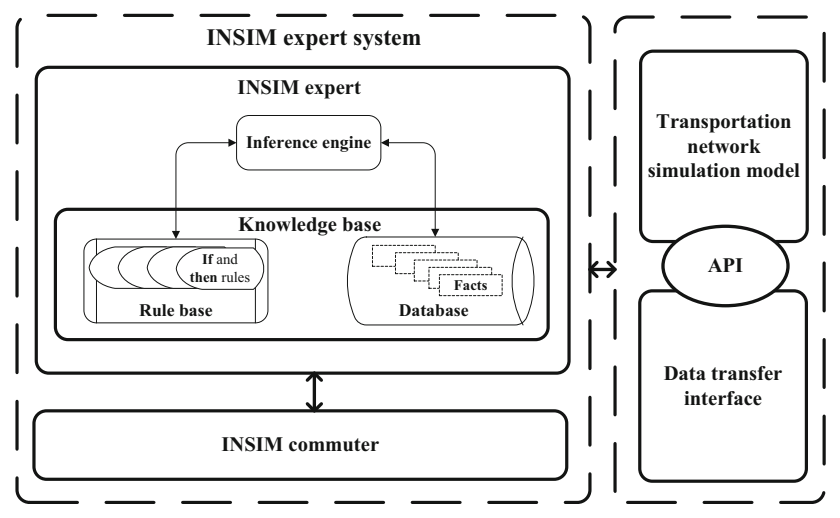

Fig. 1 Architecture of the INSIM

Currently, the simulation models are not sufficiently capable such that all the existing ITS components can be evaluated. Furthermore, in many modelling applications a more disaggregated approach to model socio-economic processes is generally desirable to reduce the model aggregation [17-21]. These two observations together have led inevitably to the adoption of a sophisticated microsimulation platform to model the ITS systems in an intelligent environment, where the simulation is closer to reality based on the production-rule system approach. The premise underlying a production-rule system is that choice behaviour in a certain context can be better described by a series of "if-then" rules. If the condition in the "if" part is true, then the action of the "then" part follows. This approach utilises an external element (agent) that is integrated with the system to rationalise the "if-then" process within the system. Such an approach is known as the agent-based simulation and has been shown to have the potential to model complicated cognitive processes. This is the approach taken in order to fill the gap of existing simulation research into the impact of IMTI system, the authors have developed a rule-based mode choice model, the intelligent network simulation model (INSIM), as reported in an earlier study [22]. The INSIM possesses the sophistication to imitate the commuters' mode choice behaviour in an information-rich environment.

INSIM has four main components: INSIM expert system (IES), transportation network simulation model (based on PARAMICS), application programming interface (API), and data transfer interface (DTI), as shown in Fig. 1. The IES is responsible for generating mode choice decision. The transportation network simulation model simulates the traffic and generates travel statistics as output files. These output text files are collected by the API, and transformed to Microsoft Access Database (MDB) files and exported to DTI, which is a database that stores the PARAMICS output files in MDB format while retaining intact the original time-dependent updated origin and destination (OD) matrix file. The API takes in the information in the OD matrix file and releases the vehicles in the simulated network according to the pattern specified in the file. The IES is designed in a way to provide the flexibility to adopt an alternative mode choice model that can give prediction of commuters' mode choice. Therefore, the impacts of realtime IMTI on the overall performance of the transportation network can be analysed.

In most current research on intelligent network simulation model, a risk strategy is typically adopted to handle the risk of network uncertainty [23-28]. In the similar manner, the most important characteristics of the IES are its highquality performance and adaptive learning. The highquality performance assures system reliability and timeliness. The adaptive learning allows the IES to update dynamically and learn on its own through its experience. The reliability of the IES depends upon its decision-making process, which can be observed by two specific features, i.e. the capability to explain about the decision taken, and to deal with uncertainty. The IES is capable to explain how and why a decision was reached. The execution of rules tending towards certain decisions can be explained by the inference chain or the rule flow. Furthermore, the rule of inference has also been designed to deal with knowledge that is incomplete or not completely certain. A heuristic algorithm is developed to solve the uncertainty problem and generate a suitable decision for the provided facts. This ability of the IES allows it to provide a solution even when the provided information is not $100 \%$ accurate or is incomplete. In this regard, it is also necessary to analyse the level of accuracy of the provided solution. To check the accuracy of the designed algorithm, an experiment was conducted and its level of mode prediction accuracy was analysed and has been reported in an earlier study by the Authors [22].

Accurate simulation results are highly dependent on the selection of model parameters and the calibration methodology [29, 30]. Calibration studies have been performed for various traffic simulation tools [31-33]. The typical calibration method is to minimise the differences between the observed data and simulation output during a certain time period on a typical day. Before application of INSIM, each module in INSIM is to be well calibrated with real traffic data to ensure realistic simulation results. This paper covers the important task involved in the calibration of the INSIM, especially IES, and is organised as follows. The study network and network coding are presented in Sect. 2. Detailed discussions regarding the calibration of INSIM are then provided in Sect. 3 and followed by conclusions in Sect. 4. 


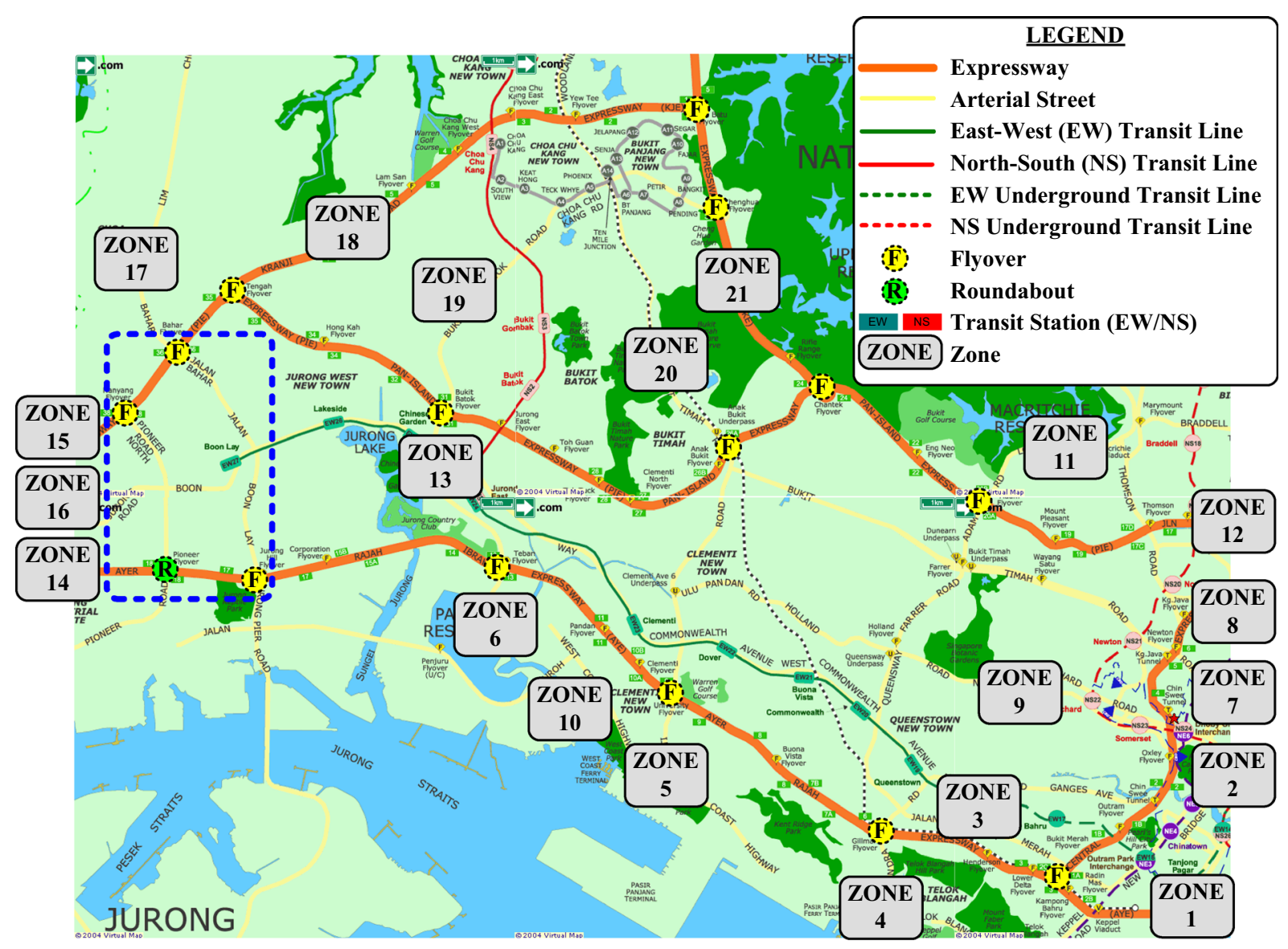

Fig. 2 Overall view of the simulated transportation network (source: Onemap, Singapore)

\section{Study network}

To assess the capabilities of the integrated IES with the transportation network simulation model, the transportation network for the central and western part of Singapore was selected for the simulation. The simulated network covers the area bounded by 5 major expressways and numerous arterial streets. The expressways are Pan-Island Expressway $(18 \mathrm{~km})$, Ayer Rajah Expressway $(19 \mathrm{~km})$, Central Expressway (9 km), Bukit Timah Expressway (5 km), and Kranji Expressway $(9 \mathrm{~km})$. In the multimodal network established for this study, there are a total of 21 residential and commercial zones, 2 Mass Rapid Transit (MRT) Lines, 23 MRT stations, 2 MRT interchanges, 38 selected signalised road junctions, 14 flyovers, and 1 roundabout. Every MRT station has feeder bus services, which traverse between nearby zones and MRT stations. Figure 2 shows the overall view of the simulated multimodal transportation network.

The physical data of the selected transportation network were collected through a survey of the study area, and also from several reliable web-based sources. All the selected expressways as well as two arterial streets (i.e. Jalan Boon Lay and Pioneer North Road) were covered. The transport network design criteria provided by the Land Transport Authority (LTA) of Singapore [34] was also consulted. The road geometrics and details of the signalised junctions were observed and sketched during site surveys. The details of public mode of transport were collected from Singapore Mass Rapid Transit's website [35].

\subsection{Geometric design}

The imported map of the study area provided all the essential details regarding the road network layout, location of flyovers, roundabouts and road junctions, MRT network, MRT stations, and bus interchanges. It also gave the details about residential, recreational, and commercial zones. This map was loaded and displayed as the background, over which nodes, links, bus stops, and MRT stations were placed.

The expressways are mostly 3-lane dual carriageways, except at certain locations where they are upgraded to 4-lane dual carriageways. The expressways have on-ramps 
and slip lanes that allow the vehicles to enter or exit, respectively. All the arterial roads are 2-lane dual carriageways. The lane widths are $3.7 \mathrm{~m}$ for expressways and $3.4 \mathrm{~m}$ for other roads. The speed limits vary from 80 to $90 \mathrm{~km} / \mathrm{h}$ for expressways and 60 to $70 \mathrm{~km} / \mathrm{h}$ for arterials roads.

The road network in PARAMICS was coded according to the road geometrics, including the junctions, roundabouts, and flyovers. The type of links for expressways and arterial streets were specifically designed and provided in the "category" file, where details about number of lanes and speed limits were defined. The speed limit for expressways was standardised at $85 \mathrm{~km} / \mathrm{h}$ and for arterial streets at $55 \mathrm{~km} / \mathrm{h}$, given that PARAMICS models simulate the average travelling speed at $10 \mathrm{~km} / \mathrm{h}$ higher than the defined values.

\subsection{Surveillance equipment}

In the real network, traffic streams are being routinely observed via video cameras as well as loop detectors, and the statistics regarding traffic volumes and speeds are estimated and provided to certain users through web-based services. The data for the selected network were extracted from the archived database of such records.

To collect the simulated traffic statistics, 18 loop detectors were coded at points where surveillance cameras and loop detectors were present in the physical network. The loop detectors provided information about traffic counts and average travelling speeds on specific links. The gathered traffic data were also utilised in the calibration process of the simulated transportation network model.

\subsection{Signalised junctions}

A total of 38 signalised junctions were selected for the simulation model. These junctions were either cross-junctions or T-junctions, with slip lanes for left turning vehicles. The signal phasing patterns are fixed with varying signal cycle lengths. All the signalised junctions have pedestrian cross-walks. The signal phasing pattern is such that there are $3 \mathrm{~s}$ amber time and $2 \mathrm{~s}$ all-red time. The maximum cycle time is $120 \mathrm{~s}$ and maximum green time of any approach is $50 \mathrm{~s}$. The details regarding the coded phasing pattern schemes are shown in Fig. 3. The un-signalised junctions are as follows: no control (57 junctions), yield sign control (27 junctions), and stop sign control (33 junctions).

\subsection{Public mode of transport}

The public mode of transport consists of two modes: one is the bus service and the other is the MRT. It was found from

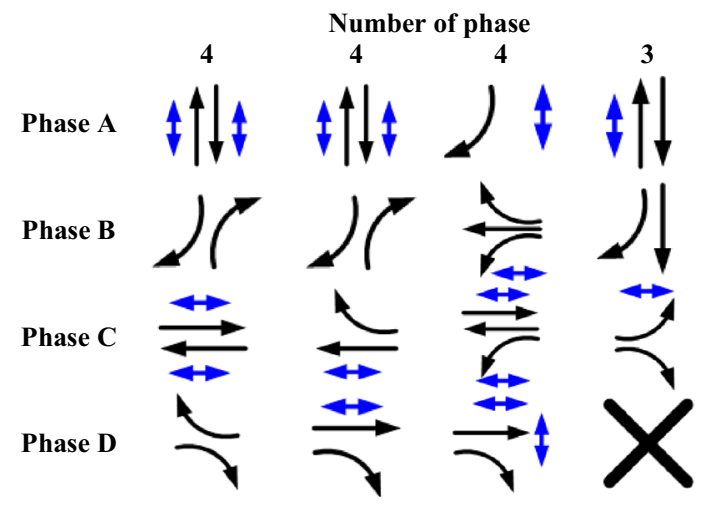

Fig. 3 Coded phasing pattern schemes

the travel behaviour survey that more than $90 \%$ of commuters in the sample reported that they had access to MRT, and they were willing to travel by public mode of transport if it provided better service [36]. One of the service criteria was the journey time, which is assumed to be lower in the case of MRT as compared to bus service. Thus, the bus service was modelled as a feeder service to MRT, and MRT was taken as the major mode of public transport for work/school trips.

The bus routes that served as feeder service to MRT and the bus stops associated to these bus routes were coded and modelled by geographical location. Similarly, the MRT lines and stations were also coded. The MRT lines were modelled as single-lane dual carriageways, with rights-ofway dedicated to MRT services only. Each MRT station was designated a zone, and explicitly simulated in such a way that the residential and commercial zones were connected to the MRT zones (stations) by feeder bus services. The simulated buses were $12 \mathrm{~m}$ long with a seating capacity of 47 passengers and a top speed of $20 \mathrm{~km} / \mathrm{h}$. The simulated trains had 6 cars with a total length of $138 \mathrm{~m}$ and a top speed of $45 \mathrm{~km} / \mathrm{h}$. Each train had a seating capacity of 372 passengers.

\subsection{Traffic information and OD matrix}

It is assumed that travellers commute in an informationrich environment, where pre-trip IMTI and en-route traffic information were available. All the driver vehicle units (DVU) were coded as controlled by familiar drivers and dynamic feedback assignment was adopted. The PARAMICS model disseminated interval traffic information to all the familiar drivers and information was updated at every minute.

An OD matrix was estimated based on the traffic counts for calibrating the developed transportation network. A traffic survey was conducted and the statistics such as traffic counts, average travelling speed, and average travel time were gathered for the calibration. 


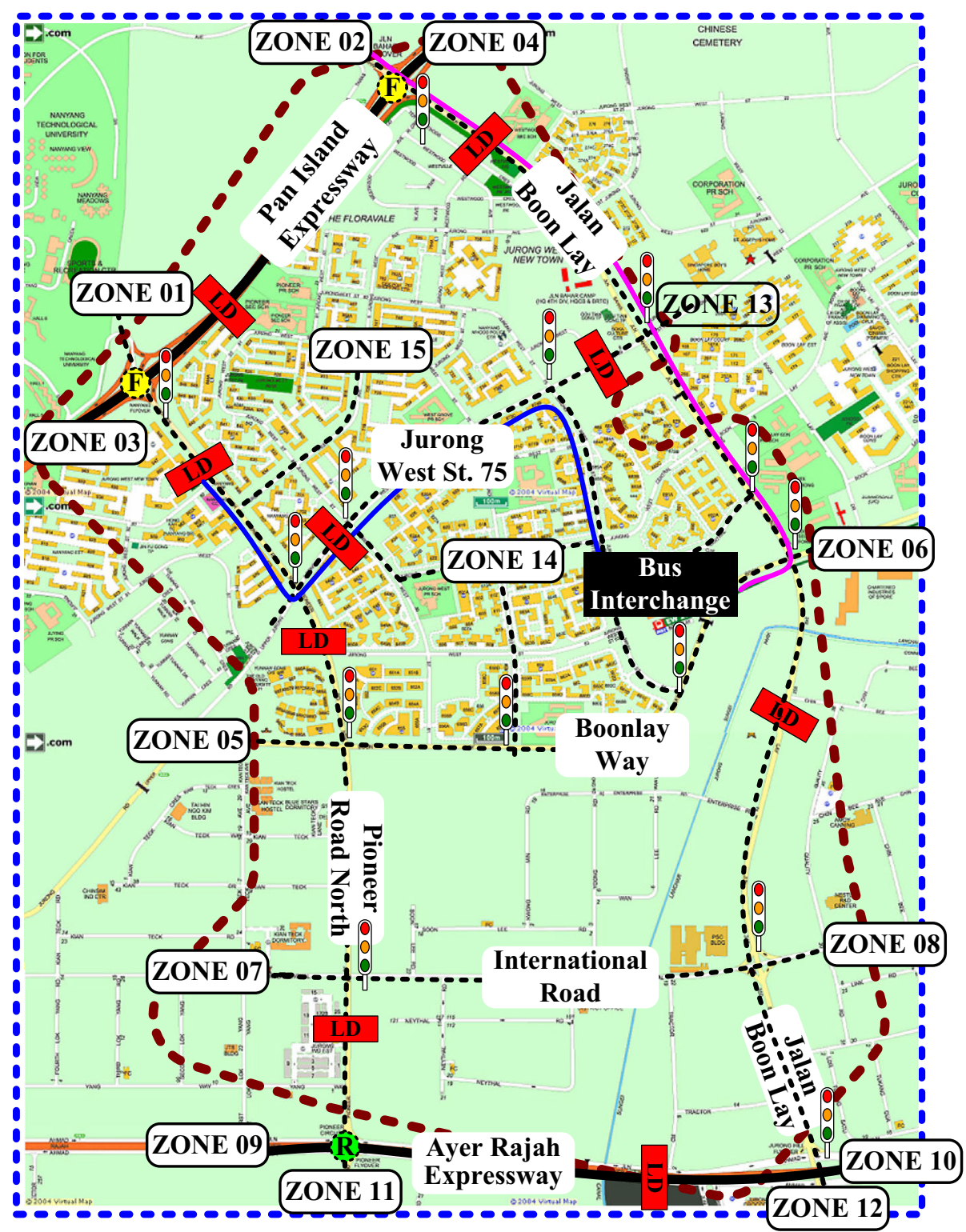

Fig. 4 Detailed map and overview of the simulated network

\section{Calibration of the INSIM network}

The calibration of the simulated network was done from two different perspectives. The first one was to adjust the traffic counts and the OD matrix in the simulation as compared with the existing situations, such that the simulated network within the complete simulation framework can be close to the real traffic conditions. The second perspective was the calibration of behavioural parameters of the PARAMICS simulation model, such that they imitated the local commuters' travel behaviour. The calibration of the behavioural parameters was necessary because the IES was developed based on the commuters' travel behaviour as observed/estimated from the travel behaviour surveys conducted in Singapore. To integrate IES with PARAMICS to simulate the local traffic environment in accordance with IES, both models should have the same behavioural assumptions, so that the behavioural parameters that govern the PARAMICS simulation model can realistically represent the behaviour of local commuters.

\subsection{Study site and basic input data}

The network chosen for the simulation model is shown in Fig. 4. To calibrate the behavioural parameters, a portion of the selected study network (marked with blue broken line) in Fig. 4, was considered. This portion of the network was selected because it covers an area bounded by two 


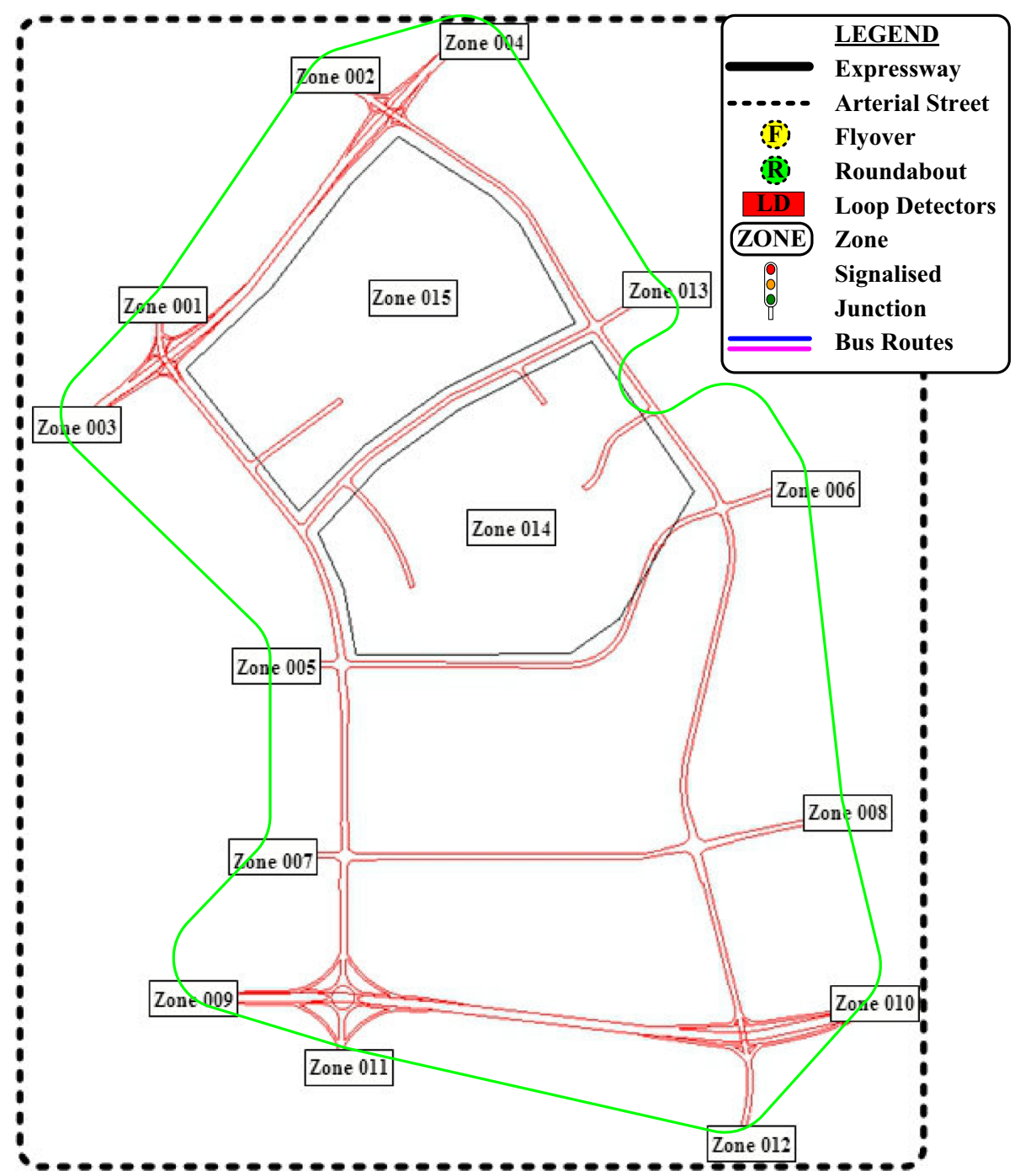

Fig. 5 Detailed simulated network showing zones, links and nodes

expressways (i.e. Pan-Island Expressway and Ayer Rajah Expressway), four arterials (i.e. Jalan Boon Lay, Boon Lay Way, International Road, Pioneer Road North), and one collector (i.e. Jurong West Street 75).

There is a major bus interchange integrated with the MRT station. Such an arrangement facilitated possible transfer between feeder buses and the transit mode. Thus, this portion of the network provided the opportunity to collect travel data for both the public and private modes, for similar trips within the same corridor. The details and overview of the simulated network are presented in Figs. 4 and 5 .

The basic input data include network geometry, driver behaviour, vehicle characteristics, transportation zones, travel demands, traffic control systems, traffic detection systems, bus routes, and bus stops. The road geometry and infrastructure locations were obtained from field surveys. The transportation zones were located at the entrance and exit nodes of all the major roads.

\subsection{Required data for calibration}

The calibration involved the checking of model results against observed data and adjusting the values of the parameters until the model results converged within an acceptable error threshold. The collected data for model calibration included traffic volume, average travelling speed, and travel time data. The traffic volume and average travelling speed data on expressways and arterial streets were obtained from an archived database. The travel time data were obtained by floating car method for Pioneer North Road and Boon Lay Way. 
To construct the real-world traffic variations in the simulation, a typical traffic variation can be represented by the traffic conditions of a typical day, of which the traffic data were the target of calibration process. The selection of a typical day can be implemented based on the comparison of traffic volume (at any selected station) of a candidate day with the average traffic volume of all candidate days using the GEH statistic, used by British engineers:

$\mathrm{GEH}=\sqrt{\frac{(E-V)^{2}}{(E+V) / 2}}$,

where $E$ is the candidate data and $V$ is the average data. If the GEH values for more than $85 \%$ of the selected stations are less than 5 , the traffic condition and the demand pattern of the candidate day are considered typical. There were 35 stations for which data were available. These stations were located on the two expressways i.e. PIE and AYE, and various arterial streets within the network. The data were collected for $1 \mathrm{~h}$ during the morning peak period from 8 to 9 AM. It was found that traffic condition on Wednesday was typical with respect to all weekdays. Therefore, the traffic volume and average travelling speed data on a certain Wednesday were chosen for calibration purpose. It was observed from the extracted data that the aggregated average traffic volume on PIE was 3600 vehicles per hour with an average travelling speed of 94 $\mathrm{km} / \mathrm{h}$, whereas on AYE the aggregated traffic volume was 5300 vehicles per hour with an average travelling speed of $87 \mathrm{~km} / \mathrm{h}$.

The travel time data were collected by floating car method. On the typical day (Wednesday) during the peak period (8-9 AM) several runs were made between Nanyang Technological University (NTU) and Boon Lay Interchange (BLI). The length of the selected route was $4.2 \mathrm{~km}$. The number of floating car runs was obtained from the following equation:

$n=\left(\frac{t s}{\mu \varepsilon} \times 100\right)^{2}$,

where $n$ is the required number of runs, $\mu$ is the mean travel time in the runs, $s$ is the standard deviation, $\varepsilon$ is the desired margin of error (percent of $\mu$ ), and $t$ (Student's t-statistic) is the confidence coefficient.

The standard deviation was unknown prior to runs being conducted. A total of 12 runs were performed. It was observed from the floating car runs that the average travel time was $314 \mathrm{~s}$ and the standard deviation was $22.3 \mathrm{~s}$. The estimated number of runs with margin of error at $5 \%$ was $9.75 \approx 10$. The $95 \%$ confidence interval at 12 runs was estimated to be $\pm 14.2 \mathrm{~s}$, and it was inferred that the true mean lies between 300 and $328 \mathrm{~s}$. The corresponding average travelling speed on this route was estimated to be between 46.1 and $50.4 \mathrm{~km} / \mathrm{h}$. The average travelling speed on the typical day for this route was observed to be $50.4 \mathrm{~km} / \mathrm{h}$ with standard deviation of $14 \mathrm{~km} / \mathrm{h}$. These results provided evidence that the estimated travel time statistics by floating car method gave the details regarding the basic travelling conditions.

Similarly, the travel time data for the public mode of transport were collected. Two bus routes were considered: the first one (SBS 199) served between NTU and Boonlay Interchange (BLI), and the other (SBS 242) served between Jurong West area and BLI. The route length of SBS 199 is $4.2 \mathrm{~km}$, and it is associated with 9 bus stops out of which 3 have bus bays. The route length of SBS 242 is $3 \mathrm{~km}$ with 13 bus stops, out of which 3 have bus bays. The frequency of SBS 199 is 4 min during peak hours and 7 min during off-peak hours and for SBS 242 is 3 min during peak hours and 5 min during off-peak hours on week-days, respectively. On each route, there are 4 signalised junctions and 2 mid-block pedestrian signals.

Initially, 30 runs were conducted on each bus route, and the travel time data were collected. For SBS 199 route, it was found that the average travelling time was $752 \mathrm{~s}$ with a standard deviation of $51.4 \mathrm{~s}$. With an error margin of $5 \%$, the estimated number of runs was $7.8 \approx 8$. The resulting $95 \%$ confidence interval was $\pm 19.5 \mathrm{~s}$ for 30 runs, which means that the true mean travel time can be between 732 and 772 s. For SBS 242 route, the average travelling time was $556 \mathrm{~s}$ and the standard deviation was $46.2 \mathrm{~s}$. With an error margin of $5 \%$, the estimated number of runs was $11.6 \approx 12$. The resulting $95 \%$ confidence interval was estimated to be $17.5 \mathrm{~s}$, which means that the true mean travel time can be in-between 538 and 574 s. The average travelling speeds based on the observed travel time values on routes SBS 199 and SBS 242 were 23.9 and $32.4 \mathrm{~km} / \mathrm{h}$, respectively.

The PARAMICS simulation model needs an OD matrix as a starting point of the calibration process. The timedependent OD matrix was generated by IES. The OD estimation process was dependent on commuters release profile and zone-based trip distribution. It is necessary that the generated OD matrix should match with some reference OD matrix, so that the PARAMICS model can simulate realistic traffic conditions. The reference OD matrix was estimated from the traffic count data obtained for the typical day.

The cordon points of the network were taken as zones, and 15-min interval traffic counts at all cordon points were gathered from the data. The total traffic attractions and generations of each zone were then assumed to be the distribution of traffic with respect to the OD matrix. The available loop detector data were then compared for checking the network-wide equilibrium of traffic volumes, such that the traffic entering the network should be equal to 
the traffic leaving the network. To estimate the OD matrix, the observed traffic counts at the cordon points were taken into consideration. These traffic counts were assumed to be the total attraction and generation from the respective zones corresponding to the cordon points. Once the traffic volume being generated and attracted to each zone was estimated, it was then compared with a reference OD matrix. This reference OD matrix was obtained from the study done by Ang [37], which provided the details regarding the traffic volumes being generated during the morning peak period.

The data gathered from the traffic survey provided details about the traffic volumes for 7 zones. The traffic volume data for these zones were compared with those provided in the reference OD matrix and the growth factor was estimated. Based on the estimated growth factor, which was found to be $5.69 \%$ per annum, the total productions and attractions for each zone of the reference OD matrix were revised. The adopted expansion procedure was

(a) the total production/attraction for each zone of the reference OD was expanded, excluding the total productions/attractions for the zones that were obtained from the traffic survey;

(b) all the expanded total productions/attractions were summed up, and compared with the expanded total traffic volume, to estimate the overall difference,

(c) the estimated overall difference was distributed among all the zone-based total productions/attractions depending upon their share of traffic volume aggregated in the total traffic volume;

(d) each zone-based total productions/attractions were then adjusted according to the allocated share of the estimated difference;

(e) the adjusted zone-based total productions/attractions were again summed up, such that their sum was now equal to the expanded total traffic volume; and

(f) the finalised zone-based total productions/attractions were taken as the target values to be used in the final version of the estimated OD matrix.

Each cell value of the reference OD matrix was also expanded, corresponding to the expanded total traffic volume. The zone-based total productions/attractions for these cell values were estimated and taken as the base case values. The Furness technique was then used for balancing the base case values with the target values of the zonebased total productions/attractions. Only the expanded values of the zone-based total productions/attractions were changed, and the total productions/attractions estimated from the traffic survey were unchanged. Based on the estimated OD matrix, the percentages of trips being productions and attractions for each zone were calculated. The zone-based distribution of trips was estimated and is presented in Table 1. The zone-based trip distribution was utilised by IES to generate the time-dependent OD matrix.

\subsection{Preliminary testing}

In INSIM, three types of demand-related data are necessary: zone-based percentile distribution of trips, percentage of total traffic flow to be released onto the network in each time interval, and the total number of trips to be generated. Field data were processed to gather the demand-related data along with the required performance measures. The transportation network model was simulated based on the demand-related data, and was calibrated by comparing the simulated output results with the gathered performance measures. It was observed from the field data that on a typical day the peak period was from 8 to $9 \mathrm{AM}$, and 33,120 private mode trips were generated within the physical geography of the simulated network during this period. The peak period was defined by observing the traffic volumes on a typical day from 7 to 10 AM. It was found that the traffic volumes were highest during the time period of 8-9 AM. Available information in recent years [38] revealed that roughly $40 \%$ commuters took private mode for their work trip and $50 \%$ took public mode, and $10 \%$ did not require any motorised transport. Based on these statistics, a total of 79,877 trips were simulated for a 1-h time period. The estimated zone-based percentile trip distribution (Table 1), and a uniform trip release profile was also defined. Default values for vehicle population composition, vehicle characteristics, and driver aggressiveness and awareness distributions were not changed. The dynamic feedback assignment model provided by PARAMICS was adopted to replicate route choice behaviour.

Initially, 10 simulation runs were conducted in order to identify critical variables that may significantly influence the performance of PARAMICS. Variables related to driver behaviour (e.g. the distributions of aggressiveness and awareness), compositions of vehicles, driver's familiarity, etc. were examined. The number of simulation runs was based on number of vehicles (NV). The average NV value was 32,580 and the standard deviation was 335 . The estimated number of runs with a margin of error of $1 \%$ was $5.12 \approx 6$. The required simulation runs were conducted with different seed numbers. The numerical outputs of the median simulation run were then compared to corresponding real data.

Outputs used for comparison were related to the total demand generated and the total flow reaching the destination. The outputs were aggregated and analysed using Microsoft Excel. Outputs aggregated in this step for further comparison included total generations (total vehicles released in $1 \mathrm{~h}$ ), and total attractions (total number of 
Table 1 Zone-based trip distribution (\%)

\begin{tabular}{|c|c|c|c|c|c|c|c|c|c|c|c|c|c|c|c|c|c|c|}
\hline $\mathrm{O} / \mathrm{D}$ & 1 & 2 & 3 & 4 & 5 & 6 & 7 & 8 & 9 & 10 & 11 & 12 & 21 & 22 & 23 & 24 & 25 & 26 \\
\hline 1 & & 0.1 & 1 & 0.2 & 0.3 & & 0.2 & & 0.1 & 0.4 & 0 & 0.5 & 0.2 & 0.2 & 0.6 & & 0.1 & 0.7 \\
\hline 2 & 0 & & 0.3 & 0.1 & 0.2 & & 0 & & 0 & 0.1 & & 0.1 & 0.1 & 0.1 & 0.1 & & 0.1 & \\
\hline 3 & 0.6 & 0.3 & & 0.8 & 2.1 & 0.2 & 0.3 & 0.8 & 0.6 & 0.7 & 0.2 & 0.5 & 0.2 & 0.5 & 0.8 & & 0.3 & 0.1 \\
\hline 4 & 0.1 & 0.1 & 0.7 & & 0.3 & & 0 & 0.2 & 0.1 & 0.3 & 0.1 & 0.1 & 0.1 & 0.1 & 0.2 & & 0.1 & 0.2 \\
\hline 5 & 0.2 & 0.3 & 2 & 0.3 & & 0.1 & 0.1 & 0.7 & 0.4 & 1.1 & 0.1 & 0.4 & 0.8 & 0.6 & 1.4 & 0 & 0.1 & 0.2 \\
\hline 6 & & & 0.2 & & 0.1 & & 0 & & 0 & 0.2 & 0 & 0.2 & 0.2 & 0 & 0.2 & & 0.1 & 0.1 \\
\hline 7 & 0.1 & & 0.4 & 0 & 0.1 & 0 & & 0.3 & 0.1 & 0.2 & 0 & 0.4 & 0.1 & 0.1 & 0.2 & & 0.1 & 0.3 \\
\hline 8 & 0.1 & & 1.5 & 0.3 & 0.4 & & 0.5 & & 0.1 & 1.4 & 0.1 & 2.1 & 0.8 & 0.3 & 1.4 & & 0.2 & \\
\hline 9 & 0.1 & 0 & 0.8 & 0.1 & 0.5 & 0 & 0 & 0.1 & & 0.8 & 0.1 & 0.6 & 0.4 & 0.2 & 0.9 & & 0.2 & 0.6 \\
\hline 10 & 0.2 & 0.2 & 0.7 & 0.3 & 1 & 0.2 & 0.2 & 0.6 & 0.8 & & 0.2 & 0.4 & 0.7 & 0.3 & 0.5 & & 0.1 & 0.1 \\
\hline 11 & 0 & & 0.1 & 0.1 & 0 & 0 & 0 & 0.3 & 0.1 & 0.2 & & 0.1 & 0.1 & 0 & 0.1 & & 0 & 0.2 \\
\hline 12 & 0.4 & 0.2 & 0.7 & 0.3 & 0.9 & 0.2 & 0.5 & 3 & 0.9 & 0.6 & 0.2 & & 0.2 & 0.3 & 0.6 & & 0.6 & 0.8 \\
\hline 21 & 0.1 & 0 & 0.1 & 0.1 & 0.8 & 0 & 0.1 & 0.7 & 0.3 & 0.6 & 0.1 & 0.1 & & 0.1 & 0.5 & & 0 & 0.1 \\
\hline 22 & 0.3 & 0.2 & 1.9 & 0.3 & 2.3 & 0.1 & 0.1 & 0.7 & 0.7 & 1 & 0.1 & 0.8 & 0.5 & & 3.6 & 0.1 & 0.8 & 0.3 \\
\hline 23 & 0.3 & 0.2 & 0.9 & 0.2 & 1.5 & 0.2 & 0.2 & 1.2 & 0.7 & 0.5 & 0.2 & 0.2 & 0.6 & 1.2 & & 0.1 & 0.9 & 0.2 \\
\hline 24 & & & & & 0 & & & & & & & & & 0.1 & 0.1 & & & \\
\hline 25 & 0.1 & 0.1 & 0.4 & 0 & 0.2 & 0.1 & 0.1 & & 0.2 & 0.3 & 0 & 0.5 & 0.1 & 0.3 & 1.2 & & & 0.2 \\
\hline 26 & 1 & & 0.2 & 0.3 & 0.5 & 0.6 & 0.9 & & 0.7 & & 0.4 & 1.2 & & 0.2 & 0.4 & & 0.6 & \\
\hline
\end{tabular}

Empty cells denote ZERO value

Table 2 Output data comparison with the un-calibrated model

\begin{tabular}{llll}
\hline & Simulated & Actual & Error (\%) \\
\hline Total demand (production) & 28,782 & 33,120 & -13.09 \\
Total outflow (attraction) & 25,288 & 33,120 & -23.64 \\
\hline
\end{tabular}

vehicles completing their journey in $1 \mathrm{~h}$ ). Results in Table 2 indicate a shortage in the number of vehicles being released into the network, and the number of vehicles reaching their destinations. The difference in productions and attractions is due to vehicles which were released in the network but have not reached their destinations. Thus, the difference between the productions and attractions is equal to the total number of vehicles which are still in the network. Therefore, parameter values other than the default needed to be explored. In order to improve the accuracy of the simulation at this stage, the types of vehicles using the network and their respective percentages in the vehicle population were adjusted. There was no systematic way for adjusting the percentage of different types of vehicles except to experiment different combinations so as to get acceptable results. After several combinations the final percentages of different types of vehicles used in the simulation are presented in Table 3.

It was observed during the simulation that the DVU traversing on expressways did not use the outermost lanes, specifically at the merging points at on-ramps along expressway lanes. At such locations, the vehicles slowed
Table 3 Calibrated percentages of different types of vehicles

\begin{tabular}{llc}
\hline Type & & Percentage \\
\hline Serial number & Shape & \\
\hline 1 & Private car & 50 \\
2 & Taxi & 15 \\
3 & LGV & 9 \\
4 & OGV 1 & 8 \\
5 & OGV 2 & 8 \\
6 & Mini bus & 7 \\
7 & Coach & 3 \\
Total & & 100 \\
\hline
\end{tabular}

down for longer time periods, and such behaviour resulted in heavy congestion. To analyse such issue, three parameters concerned with driver behaviour were investigated. Two parameter sets in particular, aggressiveness and awareness, were examined-since these two significantly influence the behaviour of drivers. There are four default types of distributions associated with each of these two parameters. Numerous runs with different combinations of distributions were conducted until the outputs of lane use were improved further. The third parameter was the ramp awareness distance. It was observed from the PARAMICS graphical interface of the simulated network that increasing the length of ramp awareness distance increased the flow on expressways and improved the outermost lane utilisation. The ramp awareness distance was adjusted one by one 
for all the ramps, and the minimum value was adjusted to $150 \mathrm{~m}$. Using combination of the above adjustments, 10 simulation runs were conducted and the improved results are summarised in Table 4.

Theoretically, it is a dynamic OD demand estimation problem. To date, there are some OD estimation tools e.g. Estimator of PARAMICS, but their capabilities and potentials for a corridor network are still unclear [39]. The time-dependent OD matrix was estimated on the basis of zone-based percentile trip distribution. The proposed dynamic OD estimation process could be regarded as a process that assigns the total OD to a series of consecutive time stamps. The time-dependent OD demand for each time stamp was then extracted by reconstructing the dynamic OD matrix based on a set of demand profiles for every time stamp, e.g. 1 or $5 \mathrm{~min}$. INSIM allows the user to define the time-dependent releases of demand (trips) and the zone-based trip distribution.

The field data provided 15-min interval counts for all the cordon points in the network. Based on these data, the profile of vehicle production from any origin zone and that of vehicle attraction to any destination zone were thus estimated. Four different trip release profiles for different time stamps were developed as shown in Fig. 6, such that:

(a) A uniform distribution was assumed for the demand release profile, i.e. RP1. The total demand was uniformly spread over $60 \mathrm{~min}$ of the simulation period with a time stamp value of $5 \mathrm{~min}$. For the first four time stamps, $9 \%$ of the total volume was

Table 4 Output data comparison based on the calibrated model

\begin{tabular}{llll}
\hline & Simulated & Actual & Error $(\%)$ \\
\hline Total demand (generation) & 32,428 & 33,120 & -2.09 \\
Total outflow (attraction) & 31,914 & 33,120 & -3.64 \\
\hline
\end{tabular}

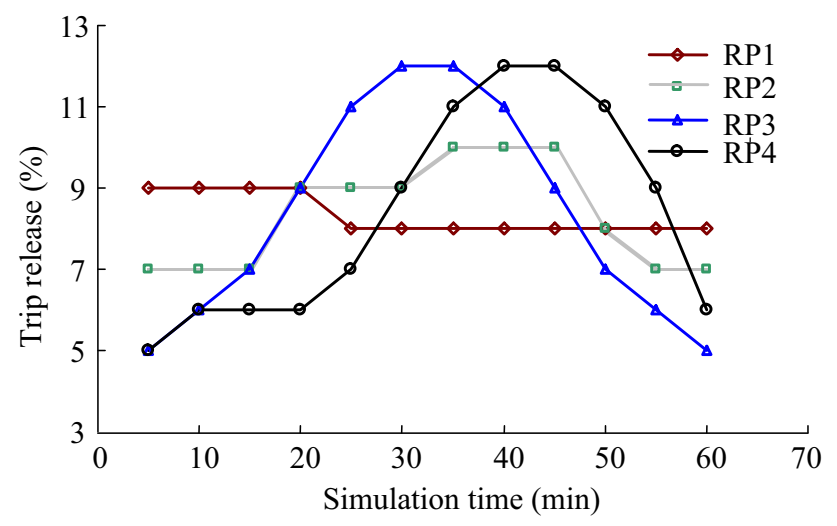

Fig. 6 Different trips release profiles released, and in the remaining 8 time stamps $8 \%$ were released.

(b) A nonuniform distribution was assumed for the demand profile RP2. The 15-min counts were segregated into 5-min counts, by uniformly distributing the 15-min counts over 3 periods of 5-min duration, such that $7 \%$ of demand was released in each 5-min time stamp for the first 15-min time interval, $9 \%$ of demand was released in each 5-min time stamp for second 15-min time interval, and so on.

(c) A normal distribution was assumed for the demand profile RP3, such that the maximum release of trips occurred during the 2nd and 3rd quarters of the simulation period. In this profile the demand increased uniformly and arrived at the peak discharge of $12 \%$, after which it decreased uniformly.

(d) A normal distribution skewed towards the right was assumed for the demand profile RP4. In this profile the peak discharge of $12 \%$ occurred in the $3 \mathrm{rd}$ quarter of the simulation period. This profile imitated the real traffic flow condition.

\subsection{Calibration of route choice model}

In the OD estimation process, the network, traffic and route choice behaviour parameters were to be fixed initially, as this process is based on the traffic assignment matrix that is affected by any change in the simulation input parameters.

Due to the existence of expressways and parallel streets in the study network, the routing algorithm adopted in the PARAMICS simulation was important. The network was calibrated using the dynamic feedback assignment model provided by PARAMICS. Dynamic feedback assignment in PARAMICS assumes that different drivers perceive different costs from a decision node to the destination. The perceived cost was calculated, and the perceived shortest route was chosen at the decision node. At this stage the parameter to be calibrated for the route choice model is the number of drivers who are familiar with the road network. Since there was no data to calibrate it, it was assumed that most drivers in the morning peak period were familiar drivers who had the knowledge of road network and traffic conditions. Therefore, it was assumed that $95 \%$ of drivers were familiar drivers, who could choose their route from the available options.

\subsection{Secondary testing}

Multiple simulation runs for each parameter combined with the each trip release profile were also performed. During these simulations, certain changes in the cell values (i.e. specific origin to destination traffic volumes) of the 
Table 5 Traffic count calibration results for release profile RP4

\begin{tabular}{|c|c|c|c|c|c|c|c|c|c|c|c|c|}
\hline \multirow[t]{2}{*}{ ID } & \multicolumn{3}{|c|}{ Traffic count $(0800-0815)$} & \multicolumn{3}{|c|}{ Traffic count (0815-0830) } & \multicolumn{3}{|c|}{ Traffic count $(0830-0845)$} & \multicolumn{3}{|c|}{ Traffic count $(0845-0900)$} \\
\hline & Act & Sim & GEH & Act & Sim & GEH & Act & Sim & GEH & Act & Sim & GEH \\
\hline 02851 & 793 & 778 & 0.54 & 947 & 875 & 2.39 & 961 & 1037 & 2.4 & 853 & 870 & 0.58 \\
\hline 06958 & 161 & 158 & 0.24 & 192 & 193 & 0.07 & 195 & 231 & 2.47 & 173 & 200 & 1.98 \\
\hline 06966 & 68 & 57 & 1.39 & 81 & 71 & 1.15 & 82 & 68 & 1.62 & 73 & 82 & 1.02 \\
\hline 06967 & 122 & 96 & 2.49 & 146 & 115 & 2.71 & 148 & 118 & 2.6 & 131 & 137 & 0.52 \\
\hline 06975 & 575 & 564 & 0.46 & 687 & 674 & 0.5 & 697 & 701 & 0.15 & 619 & 581 & 1.55 \\
\hline 07818 & 219 & 195 & 1.67 & 262 & 268 & 0.37 & 265 & 250 & 0.93 & 236 & 176 & 4.18 \\
\hline 07819 & 194 & 149 & 3.44 & 232 & 183 & 3.4 & 235 & 184 & 3.52 & 209 & 252 & 2.83 \\
\hline 07822 & 192 & 144 & 3.7 & 229 & 276 & 2.96 & 233 & 203 & 2.03 & 207 & 210 & 0.21 \\
\hline 07823 & 140 & 145 & 0.42 & 167 & 117 & 4.2 & 170 & 124 & 3.79 & 151 & 181 & 2.33 \\
\hline 07842 & 234 & 244 & 0.65 & 279 & 330 & 2.92 & 284 & 292 & 0.47 & 252 & 266 & 0.87 \\
\hline 07843 & 192 & 156 & 2.73 & 229 & 252 & 1.48 & 233 & 323 & 5.4 & 207 & 283 & 4.86 \\
\hline 10570 & 73 & 64 & 1.09 & 87 & 93 & 0.63 & 88 & 101 & 1.34 & 79 & 82 & 0.33 \\
\hline 10571 & 219 & 173 & 3.29 & 262 & 287 & 1.51 & 265 & 306 & 2.43 & 236 & 187 & 3.37 \\
\hline 10574 & 170 & 97 & 6.32 & 203 & 214 & 0.76 & 206 & 255 & 3.23 & 183 & 183 & 0 \\
\hline 10578 & 176 & 131 & 3.63 & 210 & 162 & 3.52 & 213 & 236 & 1.54 & 189 & 193 & 0.29 \\
\hline 10579 & 315 & 252 & 3.74 & 376 & 359 & 0.89 & 382 & 395 & 0.66 & 339 & 323 & 0.88 \\
\hline 10586 & 648 & 592 & 2.25 & 774 & 797 & 0.82 & 785 & 849 & 2.24 & 697 & 745 & 1.79 \\
\hline 10587 & 352 & 321 & 1.69 & 420 & 475 & 2.6 & 427 & 550 & 5.57 & 379 & 386 & 0.36 \\
\hline
\end{tabular}

Act actual, Sim simulation

estimated OD matrix were incorporated. After multiple iterations, the calibration criteria were satisfied. The highest GEH value was obtained by adopting RP4 trip release profiles with $95 \%$ of familiar drivers. The calibration results of this step are shown in Table 5, which presents the 15-min intervals traffic counts at the selected measurement stations. It shows that for more than $87.5 \%$ of all measurement locations, their GEH values were lower than 5 , which satisfied the calibration acceptance criterion of this step.

\subsection{Calibration of driving behaviour model}

The last step was to calibrate the driver behaviour model to reflect local driver characteristics. The local driver characteristics can be examined through the comparison of the simulated and observed volume-occupancy curves drawn based on aggregated point detector data, and the point-topoint travel time measurements. Thus, the network was further calibrated under the following conditions: the network has been coded and partially calibrated for the parameters in above section; there was no data that can support the calibration of route choice model; the dynamic assignment model in the microscopic simulator can be accepted; and the zone-based percentile trip distribution and trip release profile have been obtained.
The objective function is to minimise the deviations among the observed and the simulated volume-occupancy curves, and the point-to-point travel time measurements, subject to the condition that the traffic counts, which have already been calibrated, do not change. But if the traffic counts do change, then they must satisfy the GEH calibration acceptance criteria. The point-to-point travel time match was performed only for one route between NTU and Boon Lay Interchange (BLI). The driver behaviour model can be calibrated by adjusting the car-following and lanechanging models. The global parameters for the car-following and lane-changing models are mean headway and driver reaction time, which can drastically influence the simulated driver behaviour. These two parameters were fine-tuned by trial-and-error. The purpose was to reconstruct traffic variations and match the congestion pattern of the study network, so that the resulting simulated travel time measurement matched with the real life estimated travel time values. Five different combinations of mean headway and driver reaction time were analysed. For each combination 5 simulation runs were performed and the median NV value was taken into consideration. It was observed that higher values of mean headway and reaction time resulted in lower travelling speeds and vice versa.

The final calibrated mean target headway and driver reaction time were 0.85 and 0.75 , respectively. The 
calibrated demand profile for the simulated peak period is shown in Fig. 7.

The root mean square percent error (RMSP) was estimated to examine the error between the simulated traffic demand and the actual observed traffic demand, and it was found to be $8.29 \%$. Figure 8 shows the comparison of observed and simulated point-to-point travel time for the selected route, which has an RMSP value of $2.49 \%$. Based on the lower value of RMSP, it can be stated that calibration process has been effective as indicated by the test statistics.

\subsection{Calibration of public mode of transport}

The last stage of calibration process was to calibrate the public mode of transport. Two public modes of transport i.e. train and bus services were simulated. As the bus shares the right-of-way on the surface street network, its journey time can be affected by the congestion occurring in the network. The bus journey time is dependent on two

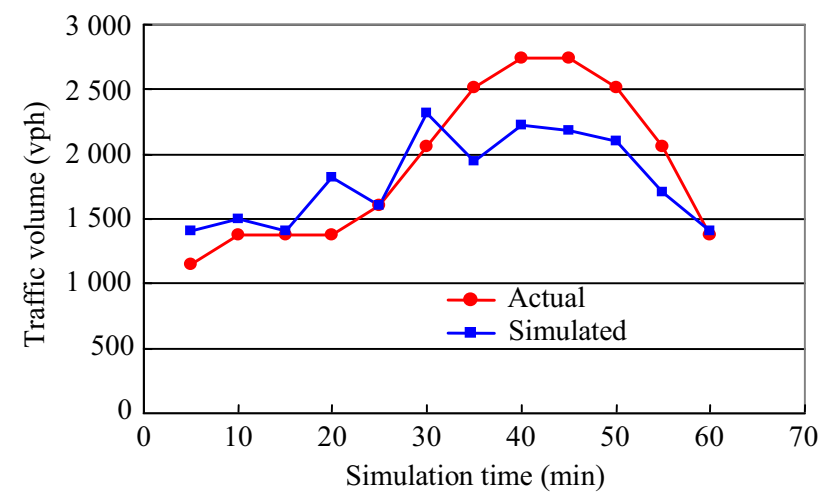

Fig. 7 Calibrated demand profile

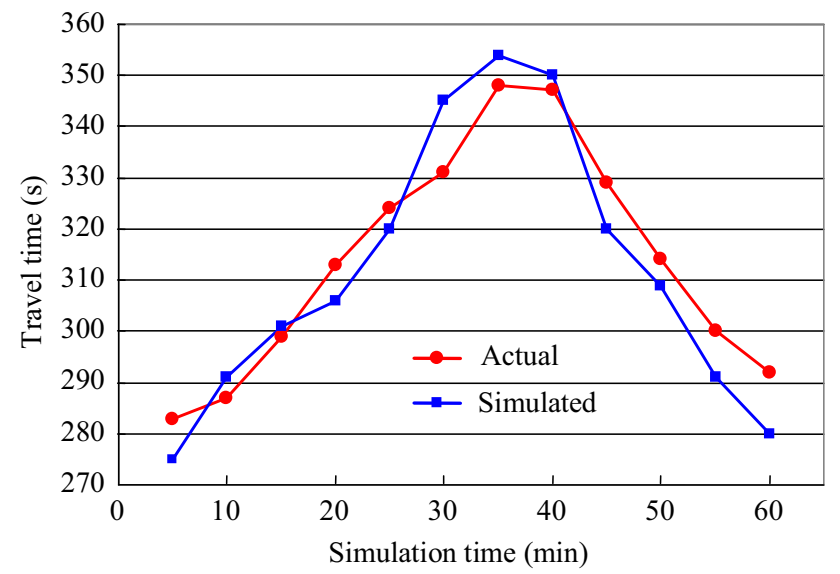

Fig. 8 Observed and simulated point-to-point travel time parameters namely the average travelling speed of buses and the number of passengers waiting at bus stops.

The number of waiting passengers can influence the travel time by affecting the bus dwell time at the bus stops, which is an integral part of travel time. Increasing the number of passengers can increase the boarding passengers' queue length resulting in longer bus dwell time at the bus stops and vice versa. Thus, the bus dwell time can be a function of number of passengers arriving at the bus stop. The public mode of transport was calibrated by adjusting the bus travel time. The selected parameters were the average travelling speed by bus and the arrival rate of passengers at the bus stop. The objective function was to minimise the difference between the simulated and observed bus travel times. The field data were available for two bus routes, i.e. SBS 242 and SBS 199. Along with other bus services these two routes were simulated.

Initially the bus travel time was calibrated by adjusting the travelling speed. Three different speeds, i.e. 15, 20, and $25 \mathrm{~km} / \mathrm{h}$, were simulated. Each speed was simulated for 5 times with different seed number. Then the bus travel time

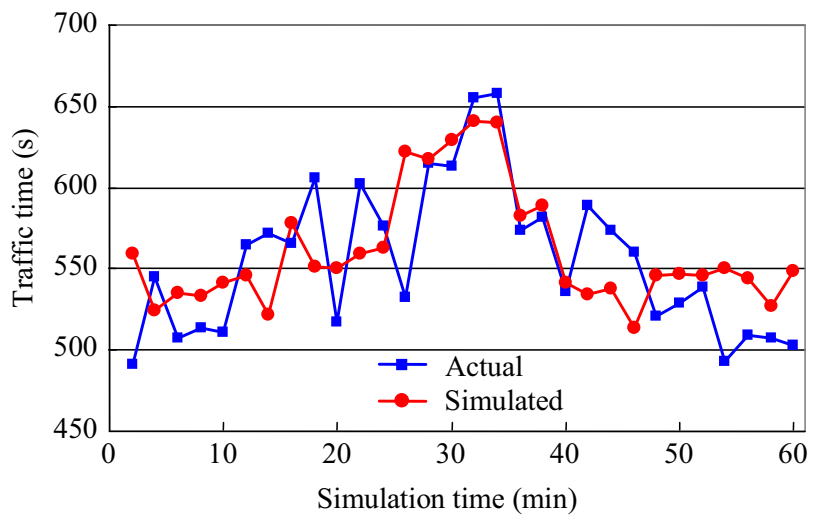

Fig. 9 Observed and simulated travel time by bus route SBS 242

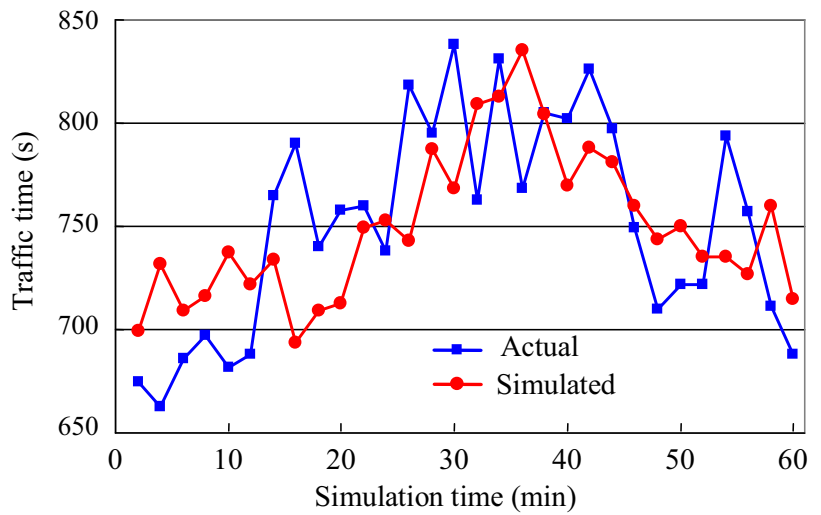

Fig. 10 Observed and simulated travel time by bus route SBS 199 
was fine-tuned by adjusting the arrival rate of passengers at each bus stop. After multiple simulation runs, the bus journey time was found to be acceptable. The average travel time measured for buses from simulation were compared with the average travel time for buses measured in the field. The comparison of observed and simulated point-to-point travel times on bus route SBS 242 and SBS 199 are presented in Figs. 9 and 10, respectively. The RMSP was estimated to be $9.54 \%$ for SBS 242, and $9.01 \%$ for SBS 199 route.

\section{Conclusion}

This paper calibrated a self-developed traffic simulation model, INSIM, with the observed field data from the central and western part of Singapore. Results reflected that the improved model and the generated outputs are acceptable. The network coding errors were corrected and a reliable time-dependent OD matrix was estimated. The INSIM was adjusted, such that the commuter release profile and the percentile trip distribution resulted in simulated traffic counts that were acceptable according to the GEH statistics. The PARAMICS route choice model was adopted and $95 \%$ of the driver population was assumed to be familiar with the road network. The driver behaviour model was calibrated, and it reflects the local driver behaviour. The mean headway and driver reaction time were adjusted to achieve the calibration of the driver behaviour model. Finally, the public mode of transport was calibrated by adjusting the average travelling speed of buses and the arrival rate of passengers at the bus stops.

Model calibration is a necessary process for various kinds of traffic simulation model, especially for newly developed rudiment before application. This paper provides the whole calibration procedure using local travel network and data. It can simulate the travel time and travelling speed parameters, which shall be utilised by the IES to generate commuters' travel mode choice under the influence of integrated traveller information, within the statistically acceptable limits, as compared to the observed data. Future tasks include applying the calibrated INSIM in various kinds of real networks to achieve its functions.

Acknowledgments This work was conducted under the $\mathrm{PhD}$ research programme of the first author (Dr Abdul Ahad Memon) at the Nanyang Technological University.

Open Access This article is distributed under the terms of the Creative Commons Attribution 4.0 International License (http:// creativecommons.org/licenses/by/4.0/), which permits unrestricted use, distribution, and reproduction in any medium, provided you give appropriate credit to the original author(s) and the source, provide a link to the Creative Commons license, and indicate if changes were made.

\section{References}

1. Chorus CG, Molin EJ, Van Wee B (2003) Use and effects of advanced traveller information services (ATIS): a review of the literature. Transp Rev 26(2):127-149

2. Tsirimpa A, Polydoropoulou A, Antoniou C (2007) Development of a mixed multinomial logit model to capture the impact of information systems on travelers' switching behavior. J Intell Transp Syst 11(2):79-89

3. Meng M, Shao CF, Zeng JJ, Dong CJ (2014) A simulation-based dynamic traffic assignment model with combined modes. PROMET-Traffic \& Transp 26(1):65-73

4. Ben-Elia E, Shiftan Y (2010) Which road do I take? A learningbased model of route-choice behavior with real-time information. Transp Res Part A 44(4):249-264

5. Emmerink P, Nijkamp P, Rietveld P (1995) Is congestion pricing a first-best strategy in transport policy? A critical review of arguments. Environ Plan B 22(5):581-602

6. Garling T, Jakobsson C, Loukopoulos P, Fujii S (2004) Adaptation of private car use in response to travel demand management measures: potential roles of intelligent transportation systems. J Intell Transp Syst 8(4):189-194

7. Lam SH, Toan T (2006) Land transport policy and public transport in Singapore. Transportation 33(2):171-188

8. Srinivasan KK, Mahmassani HS (2003) Analyzing heterogeneity and unobserved structural effects in route-switching behaviour under ATIS: a dynamic kernel logit formulation. Transp Res Part B 37(9):793-814

9. Hensher DA, Green WH (2003) The mixed logit model: the state of practice. Transportation 30(2):133-176

10. Beesley ME (1965) The value of time spent in travelling: some new evidence. Economica 32(126):174-185

11. Adler JL, Blue VJ (1988) Towards the design of intelligent traveller information systems. Transp Res Part C 6(3):157-172

12. Chen PS, Mahmassani HS (1993) Dynamic interactive simulator for studying commuter under real time information supply strategies. J Transp Res Rec 1413:12-21

13. Adler JL, Recker WW, McNally MG (1993) A systematic evaluation of the impacts of real-time traffic condition information on traffic flow. PATH Research Report, UCB-ITS-PRR-93-6, California Department of Transportation

14. Koutsopoulos HN, Lotan T, Yang Q (1994) A driving simulator and its applications for modelling route choice in the presence of information. Transp Res Part C 2(2):91-107

15. Miller J (2006) Integrated urban models: progress to date; future prospects. In: 11th international association for travel behaviour research conference, Kyoto

16. Srinivasan K (2000) Dynamic decision and adjustment processes in commuter behaviour under real time information. PhD Dissertation. The University of Texas Austin, pp. 21-26

17. Rakha HA, Van Aerde MW (1996) Comparison of simulation modules of TRANSYT and INTEGRATION Models. J Transp Res Rec 1566:1-7

18. Barceló J, Delgado M, Funes G, García D, Perarnau J, Torday A (2007) Online microscopic traffic simulation supports real-time traffic-management strategies. Proceeding of the 6th ITS European Congress, paper no. 2607

19. Jayakrishnan R, Jun-Seok O, Sahraoui A (2001) Calibration and path dynamics issues in microscopic simulation for advanced traffic management and information systems. J Transp Res Rec 1771:9-17

20. PTV AG (2001) VISSIM 3.5 User Manual. Karlsruhe

21. Wang PTR, Glassco RA (1995) Enhanced Thoreau traffic simulation for intelligent transportation systems (ITS). In: Proceedings 
of winter simulation conference, IEEE computer society, pp. $1110-1115$

22. Memon AA, Meng M, Wong YD, Lam SH (2015) Rule-based mode choice model-INSIM Expert System. J Transp Eng ASCE 141(4):04014088

23. Bell MGH, Cassir C (2002) Risk-averse user equilibrium traffic assignment: an application of game theory. Transp Res Part B 36(8):671-681

24. Chen A, Zhou Z (2010) The $\alpha$-reliable mean-excess traffic equilibrium model with stochastic travel times. Transp Res Part B 44(4):493-513

25. Lam WHK, Shao H, Sumalee A (2008) Modeling impacts of adverse weather conditions on a road network with uncertainties in demand and supply. Transp Res Part B 42(10):890-910

26. Siu B, Lo HK (2006) Doubly uncertain transport network: degradable link capacity and perception variations in traffic conditions. J Transp Res Rec 1964:59-69

27. Siu BWY, Lo HK (2008) Travel time budget in doubly uncertain transport network: degradable capacity and stochastic demand. Eur J Oper Res 191(1):166-181

28. Zhang C, Chen X, Sumalee A (2011) Robust Wardrop's user equilibrium assignment under stochastic demand and supply: expected residual minimization approach. Transp Res Part B 45(3):534-552

29. Lee JB, Ozbay K (2009) New calibration methodology for microscopic traffic simulation using enhanced simultaneous perturbation stochastic approximation approach. J Transp Res Board 2124:233-240

30. Chen XM, Wei ZH, Gao L (2011) Simulation research of traffic behavior of road intersection pedestrian. J Cent South Univ 42(s1):1037-1041
31. Balakrishna R, Antoniou C, Ben-Akiva M, Koutsopoulos HN, Wen Y (2007) Calibration of microscopic traffic simulation models: methods and application. J Transp Res Board 1999(1):198-207

32. Appiah J, Rilett LR (2010) Joint estimation of dynamic origindestination matrices and calibration of micro-simulation models using aggregate intersection turn count data. In: Transportation research board 89th annual meeting, No. 10-2764

33. Flötteröd G, Bierlaire M, Nagel K (2011) Bayesian demand calibration for dynamic traffic simulations. Trans Sci 45(4):541-561

34. Land Transport Authority. Civil design criteria for road and rail transit systems (2010). Available via fromhttp://www.lta.gov. sg/content/dam/ltaweb/corp/Industry/files/DC_EGD09106A1_ Overall.pdf

35. SMRT (2010) Network map. Available via http://www.smrt.com. sg/Trains/NetworkMap.aspx

36. Meng M, Memon AA, Wong YD, Lam SH (2015) Dynamic interactions between commuters' mode choice behaviour and integrated traveller information. PROMET-Traffic \& Transp 26(6):485-495

37. Ang SZ (2003) Surface street network simulation studies using TRANSYT and PARAMICS. M. Sc. Dissertation, School of Civil \& Environmental Engineering, Nanyang Technological University, Singapore

38. Singstat (2010) Transport characteristics. Available via http:// www.singstat.gov.sg/publications/publications_and_papers/cop2010/ census_2010_release3/cop2010sr3.pdf

39. Chu KC, Yang L, Saigal R, Saitou K (2011) Validation of stochastic traffic flow model with microscopic traffic simulation. In: Automation science and engineering (CASE), 2011 IEEE conference on, IEEE, pp. 672-677 